\title{
Las cooperativas agrarias como modelo generador de negocios con inclusión social: el caso de las cooperativas bananeras del Magdalena (Colombia)
}

Leydis Marcela Maestre Matos ${ }^{1}$, Andrea Paola Páez Cabas ${ }^{2}$, Francisco Javier Mesías ${ }^{3}$ y Jahir Lombana Coy ${ }^{4}$

Recibido: 9 de noviembre de 2018 / Aceptado: 2 de septiembre de 2019

Resumen. Las cooperativas agrarias son consideradas como un modelo organizacional que permite la mejora de las condiciones de producción y comercialización de sus productos, generando ingresos y cambios sustanciales en sus asociados. Algunas de estas cooperativas desarrollan modelos de negocios de inclusión social con los pequeños productores que la conforman. El presente artículo pretende dar a conocer cuáles son las características de las cooperativas agrarias como generadoras de negocios con inclusión de las personas menos favorecidas (lo que se conoce como la base de la pirámide) en su modelo económico. Para ello, se establecieron unas proposiciones teóricas a partir de la revisión de la literatura que fueron validadas a través del análisis de caso múltiple con una unidad de análisis simple (seis cooperativas bananeras del departamento del Magdalena, Colombia), utilizando el método deductivo. Los resultados obtenidos muestran que una cooperativa agraria desarrolla un negocio inclusivo cuando se cumplen las siguientes condiciones: hay una vinculación de pequeños productores en una cadena de valor; se genera valor económico y social para las personas involucradas; la creación y desarrollo de la cooperativa se genera por iniciativa propia de los pequeños productores, por algún actor de la cadena productiva y/o por una reglamentación establecida; y cuando el modelo cooperativo puede ser replicable y/o escalable a lo largo del tiempo.

Palabras clave: Cadena productiva; Agronegocios inclusivos; Pequeño productor; Cooperativismo; Base de la pirámide.

Claves Econlit: B55; I39; J54.

\section{[en] Agrarian cooperatives as a business model that generates social inclusion: the case of banana cooperatives of Magdalena region (Colombia)}

Abstract. Agricultural cooperatives are an organizational model to help improve the production and marketing of products. Cooperatives generate income and changes in associates. Some of these cooperatives develop business models which generate social inclusion with the small producers. The

1 Universidad del Norte, Colombia

Dirección de correo electrónico: matos1@uninorte.edu.co

2 Universidad del Norte, Colombia

Dirección de correo electrónico: andreap94c@gmail.com

3 Universidad de Extremadura, España

Dirección de correo electrónico: fjmesias@unex.es

4 Universidad del Norte, Colombia

Dirección de correo electrónico: lombanaj@uninorte.edu.co 
objective of this paper is to present the characteristics of agrarian cooperatives. These cooperatives generate businesses with economic and social inclusion of less favored people (base of the pyramid) in their economic model. To develop this research, some theoretical propositions were constructed from a literature review. These propositions were validated through a multiple case analysis (six banana cooperatives from the department of Magdalena, Colombia), using the deductive method. Results show that an agricultural cooperative develops an inclusive business when: it includes small producers in a value chain; there is generation of economic and social value; when the creation and development of the cooperative is generated by the small producers' own iniciative, by some actor of the productive chain and / or by a normativity; and when the cooperative model can be replicable and / or scalable over time.

Keywords: Productive value chain; Inclusive agribusiness; Small producer; Cooperative; Base of the pyramid.

Sumario. 1. Introducción. 2. Fundamentos teóricos 3. Metodología. 4. Resultados y discusión. 5. Conclusiones. 6. Referencias bibliográficas.

Cómo citar: Maestre Matos, L.M., Páez Cabas, A.P., Mesías, F.J. y Lombana Coy, J. (2019) Las cooperativas agrarias como modelo generador de negocios con inclusión social: el caso de las cooperativas bananeras del Magdalena (Colombia). REVESCO. Revista de Estudios Cooperativos, Tercer Cuatrimestre, № 132, pp. 195-217. DOI: 10.5209/REVE.65488.

\section{Introducción}

Para la Confederación de Cooperativas de Colombia -CONFECOOP- (2009) la palabra cooperativa surge del término cooperativismo, que relaciona la insuficiencia del hombre para solucionar aisladamente sus necesidades siendo la unión el camino para resolver sus dificultades. Zabala ${ }^{(2007)}$ por su parte, define las cooperativas como una forma de organización alternativa donde los trabajadores se unen para solventar la escases de ingresos siguiendo reglas y esquemas establecidos como principios de cooperación.

Dentro de las características fundamentales de las cooperativas están: su naturaleza participativa con fuertes lazos entre socios (Martínez, 2008), el compromiso cooperativo (Ramírez, 1999) y la dualidad organizativa (dueño y usuario). Además, la mayoría de las cooperativas cuentan con un sistema democrático particular, donde cada socio tiene derecho a un voto independientemente de la cuantía de sus aportes, por lo que las decisiones y políticas son tomadas con la participación igualitaria de todos, lo que supone la mayor diferencia entre las cooperativas y las sociedades de capital.

Las cooperativas en Colombia están regidas por el sistema de Economía Solidaria establecido por la Ley 454 de 1998 (Congreso de Colombia, 1998), el cual incluye además otras formas organizacionales como asociaciones y empresas comunitarias. Este tipo de organizaciones tienen en común el desarrollo de su actividad sin fines lucrativos, su gestión democrática participativa, ayudar a satisfacer las necesidades de los asociados y destinar sus excedentes al servicio de los asociados.

Según los datos publicados por la CONFECOOP (2017) las cooperativas son el tipo de organización empresarial con mayor número de asociados (6.131.010) en Colombia, distribuidos en 3.666 entidades, siendo las cooperativas agrarias e 
industriales las primeras del sector productivo con 259 empresas y 110.431 asociados.

Las cooperativas agrarias han sido catalogadas por varios autores como un modelo organizacional que permite la operación formal de los pequeños productores agrarios (Coraggio, 2004; Fisac et al., 2011).

Para organizaciones como CONFECOOP (2017) son una de las mejores vías para que el pequeño productor aumente sus ingresos a través de la mejora de sus condiciones de producción y comercialización. La Organización de las Naciones Unidas para la Alimentación y la Agricultura -FAO- por su parte afirma que las cooperativas constituyen un mecanismo de inclusión social agrícola que permite el desarrollo de las comunidades (FAO - Organización de las Naciones Unidas para la Alimentación y la Agricutura, 2012).

Teniendo en cuenta la relevancia presentada anteriormente, este artículo tiene como objetivo mostrar las cooperativas agrarias como modelo generador de negocios con inclusión social, que son relevantes en el ámbito empresarial ya que a través de casos de éxito de cooperativismo muestran una estrategia para aliviar la pobreza en países con altos índices de desigualdad.

\section{Fundamentos Teóricos}

Esta sección se encuentra dividida en dos partes: la primera explica el concepto de negocios inclusivos basándose en la revisión de la literatura y el segundo muestra la conceptualización de la cooperativa agraria como negocio inclusivo.

\subsection{Negocios Inclusivos}

El origen del término Negocios Inclusivos está relacionado con los trabajos realizados por Prahalad y Hart (2002) que estudiaron la situación de las personas con bajo poder adquisitivo (denominados base de la pirámide). Estos autores presentan la base de la pirámide como un mercado altamente interesante para las grandes empresas, ya que a través de la transformación de sus productos logran crear bienes con buenas características y precios bajos, aumentando la participación en el mercado y mejorando los márgenes de utilidad de las multinacionales.

Prahalad (2005) detalla el concepto de base de la pirámide mediante la ejemplificación a través de varios modelos de negocios implementados en distintos países que han alcanzado cierto nivel de éxito por atender a la población vulnerable, generando así intereses académicos y prácticas para su desarrollo.

Este concepto ha recibido críticas de autores como Kandachar y Halme (2008), Karnani (2007), London y Hart, (2010) y Simanis y Hart (2008) que afirman que el vender nuevos productos a la población perteneciente a la base de la pirámide no está contribuyendo a la mitigación de la pobreza, sino que, por el contrario, se evidencia como una oportunidad de negocio para las grandes empresas.

Por lo anterior, los autores críticos con el concepto sugieren que lo que realmente se necesita es crear estrategias que permitan a la base de la pirámide aumentar sus ingresos. Es así como surge el concepto de Negocios Inclusivos, en el 
que se observa una transición desde una estrategia netamente comercial con los consumidores pobres a la inclusión de estos dentro de la cadena de valor de una organización (Pineda, 2015).

El concepto de Negocios Inclusivos ha sido formulado principalmente por organizaciones internacionales y colombianas (Fundación AVINA, 2008; Gradl y Knobloch, 2010; PNUD - Programa de las Naciones Unidas para el Desarrollo, 2010; SNV - Servicio Holandés de Cooperación al Desarrollo y CECODES Consejo Empresarial Colombiano para el Desarrollo, 2008; SNV - Servicio Holandés de Cooperación al Desarrollo y WBCSD - Consejo Mundial Empresarial para el Desarrollo Sostenible, 2008; WBCSD - Concejo Mundial Empresarial para el Desarrollo Sostenible, 2006), mostrando que los negocios inclusivos tienen origen en la práctica empresarial de los sectores productivos.

A continuación (Tabla 1), se muestran algunas de las definiciones más relevantes que han sido utilizadas para explicar el concepto de negocios inclusivos:

Tabla. 1. Concepto de Negocios Inclusivos

\begin{tabular}{|l|l|}
\hline Concepto & Autor Institucional \\
\hline $\begin{array}{l}\text { Iniciativas económicamente rentables, } \\
\text { ambiental y socialmente responsables, que }\end{array}$ & Fundación AVINA, (2008) \\
utilizan los mecanismos del mercado para \\
mejorar la calidad de vida de personas de bajos \\
ingresos.
\end{tabular}

Fuente: Elaboración propia conforme a los autores citados.

\subsection{Características de una cooperativa agraria de pequeños productores que desarrolla negocios inclusivos}

Las cooperativas agrarias son consideradas un medio para el desarrollo de negocios con inclusión social de la base de la pirámide (Golja y Požega, 2012). Sin embargo, no todas las cooperativas agrarias con participación de pequeños productores (considerados la base de la pirámide en el sector agrícola) en la cadena productiva desarrollan negocios inclusivos. Es por ello que a continuación, se establecen las características específicas de las cooperativas agrarias que muestran inclusión social en su modelo de negocio, y que son la base de las proposiciones teóricas validadas con el estudio de casos. 
Proposición 1: los pequeños productores (base de la pirámide) deben estar incluidos en una cadena productiva.

La Corporación Financiera Internacional (2012) al definir los negocios inclusivos, menciona la incorporación de las personas con bajos ingresos en una cadena de valor, ya sea como consumidores, minoristas, proveedores o distribuidores de las operaciones básicas; haciendo parte de un negocio comercialmente viable y replicable. Por su parte, el WBCSD (2006) menciona cuatro categorías de negocios inclusivos: 1) negocios en donde los pobres actúan como compradores; 2) negocios en donde los pobres son proveedores y/o distribuidores de su cadena de valor; 3) negocios de suministro de servicios a comunidades más pobres, para la satisfacción de las necesidades básicas y 4) desarrollo impulsado por empresas grandes extractivas, que mediante su presencia ayuden a mejorar las condiciones de vida a suministradoras y comunidades cercanas.

Márquez et al. ${ }^{(2010)}$ además de apoyar las categorías mencionadas añaden que, en la segunda categoría, la base de la pirámide puede estar asociada en cooperativas.

Aplicados al sector agropecuario, los negocios inclusivos permiten involucrar a la población rural pobre como proveedores de materia prima a empresas procesadoras, comercializadoras o exportadoras de la producción agropecuaria (Van Haeringen y de Jongh, 2010).

Por otro lado, Woodhill (2016:10) muestra por primera vez el término de agronegocio inclusivo definiéndolo como "un modelo comercial, actividad o iniciativa inclusiva dentro de los sistemas de mercado agroalimentario, a escala local, nacional, regional o internacional, que busca lograr resultados favorables para los involucrados, mientras que es rentable para las entidades comerciales de la cadena". Así mismo, establece que un agronegocio es inclusivo cuando cumple una o varias de estas características (Woodhill, 2016): "1. Crean oportunidades que permiten a los pequeños agricultores y sus cooperativas convertirse en socios comerciales económicamente viables en las cadenas de suministro. 2. Apoyan a las pequeñas y medianas empresas para que prosperen como procesadores y proveedores de materia prima o servicios a lo largo de la cadena de suministro. 3 . Proporcionan oportunidades de empleo en condiciones laborales justas. 4. Establecen aglomerados / centros que ayuden a impulsar la prosperidad económica rural en general. 5. Ofrecen productos y servicios alimenticios saludables, asequibles y accesibles para los consumidores de bajos ingresos en áreas rurales y urbanas. 6. Da a todos los interesados y en particular a los grupos marginados (pequeños agricultores, mujeres, jóvenes, trabajadores no sindicados) una voz en el gobierno y la inversión". Por lo anterior, las cooperativas agrarias en donde la base de la pirámide son los pequeños agricultores se pueden categorizar en el primer tipo de agronegocio inclusivo según Woodhill (2016), cumpliendo el rol de productores de su cadena de valor ${ }^{5}$; siendo esta una condición necesaria del negocio inclusivo. Esta afirmación, es apoyada por conceptos desarrollados por el SNV - Servicio Holandés de Cooperación al Desarrollo y CECODES - Consejo Empresarial Colombiano para el Desarrollo (2008); el SNV - Servicio Holandés de

Cadena de valor: según Porter (1985) es el conjunto de actividades que generan valor al cliente final. 
Cooperación al Desarrollo y WBCSD - Consejo Mundial Empresarial para el Desarrollo Sostenible (2008) y el WBCSD - Concejo Mundial Empresarial para el Desarrollo Sostenible (2006) entre otros.

Proposición 2: Se debe generar valor económico y valor social en los involucrados.

Otra de las características propias de un negocio inclusivo es la generación de valor económico y valor social para los participantes del negocio. Algunos autores (Licandro, 2013; Fundación AVINA, 2008), asocian el valor económico con la rentabilidad del negocio en todos los eslabones de la cadena.

Inicialmente, algunos autores (Gatica et al., 2012; WBCSD - Concejo Mundial Empresarial para el Desarrollo Sostenible, 2006) hacían referencia a la generación de valor económico y social como una responsabilidad social y ambiental de las empresas hacia la base de la pirámide. Sin embargo, con la ampliación del concepto y el descubrimiento de nuevas motivaciones para la generación del negocio inclusivo, se puede incluir la autoresponsabilidad del pequeño productor para mejorar sus condiciones de vida y generar con esto emprendimientos rentables.

Woodhill (2016) es más específico en el valor económico que se puede generar en un agronegocio inclusivo a la base de la pirámide y lo relaciona con proporcionar un salario digno a los trabajadores, tener acuerdos comerciales flexibles en donde el pequeño productor tenga apoyo en suministros, pago contra reembolso, suministro de pedidos confiables y regulares, apoyo a los pequeños productores para desarrollar su poder de negociación y servicios financieros y una transparencia en la fijación de precios.

En el caso de las comercializadoras, a través de estos negocios inclusivos con pequeños productores se puede obtener como valor económico el suministro asegurado de productos agroalimentarios, lo que implica la ampliación de su oferta, el aumento en las ventas y la mejora de su imagen con la aplicación de políticas de responsabilidad social.

Con relación al valor social, en la literatura aún no hay una clara definición de su significado, sin embargo se encontró una relación del concepto con la superación personal de la base de la pirámide (Bustamante y Muñoz, 2017); mejora de su calidad de vida y construcción de capital social (Márquez et al., 2010) a través de las relaciones sociales (acceso a redes, niveles de confianza, estatus social, relaciones con instancias de gobierno, entre otros); desarrollo de habilidades (a través de capacitaciones); aumento de la autoestima; y definición de aspiraciones y acceso a información (Van Haeringen y de Jongh, 2010).

Proposición 3: El inicio del negocio inclusivo debe ser motivado y respaldado por 1) algún actor de la cadena o por 2) iniciativa de cocreación propia de la base de la pirámide.

Como se enunció anteriormente, Prahalad (2005) y su concepto de base de la pirámide fue uno de los principales autores que incentivó el trabajo de las grandes empresas hacia los más vulnerables, a través del desarrollo de productos dirigidos a satisfacer sus necesidades y su inclusión en la economía. 
Sin embargo, este trabajo de las empresas hacia la base de la pirámide se ha transformado en un proceso colaborativo de las organizaciones con la base de la pirámide en nuevos modelos de negocios inclusivos, después de críticas surgidas por diferentes académicos como Karnani (2007), Simanis y Hart (2008) y Kandachar y Halme (2008).

Es por ello que inicialmente, se ha definido como requerimiento indispensable para el desarrollo de los negocios inclusivos la relación de los nuevos emprendedores con una empresa, a la que los autores denominan "empresa ancla" (Hahn, 2012; Reficco, 2010; SNV - Servicio Holandés de Cooperación al Desarrollo y CECODES - Consejo Empresarial Colombiano para el Desarrollo, 2008). En este caso, la empresa ancla es la organización que ayuda a la creación del emprendimiento, aportando activos tangibles o intangibles al proceso (Licandro, 2013). El emprendedor es la persona o grupo de personas de la base de la pirámide que inicia y pone en funcionamiento el nuevo negocio. Para el caso de las cooperativas agrarias, el pequeño productor es quien cumple el nuevo rol de empresario (Ickis et al., 2009).

Sin embargo, el concepto desarrollado por Betancourt (2014) y apoyado por Bustamante y Muñoz (2017) menciona que para el inicio de los negocios inclusivos no se requiere en todos los casos de una empresa ancla, sino que también pueden surgir a partir de la cocreación endógena de valor propia de los pobres, con ayuda de entidades del gobierno, educativas u otro tipo de organizaciones, que permitan la inserción de los más pobres en el mercado, siendo el papel de la empresa un apoyo y no un gestor del negocio inclusivo.

\section{Proposición 4: Debe ser replicable y/o escalable a lo largo del tiempo.}

Como última característica identificada del negocio inclusivo está el poder ser un negocio replicable o escalable a lo largo del tiempo (Bustamante y Muñoz, 2017; Ishikawa y Strandberg, 2009), lo cual indica según Woodhill (2016) que puede aumentar el número de actores involucrados y/o el modelo puede ser copiado en otras cadenas de valor.

Figura. 1. Características de una cooperativa agrícola de pequeños productores que desarrolla un negocio inclusivo.

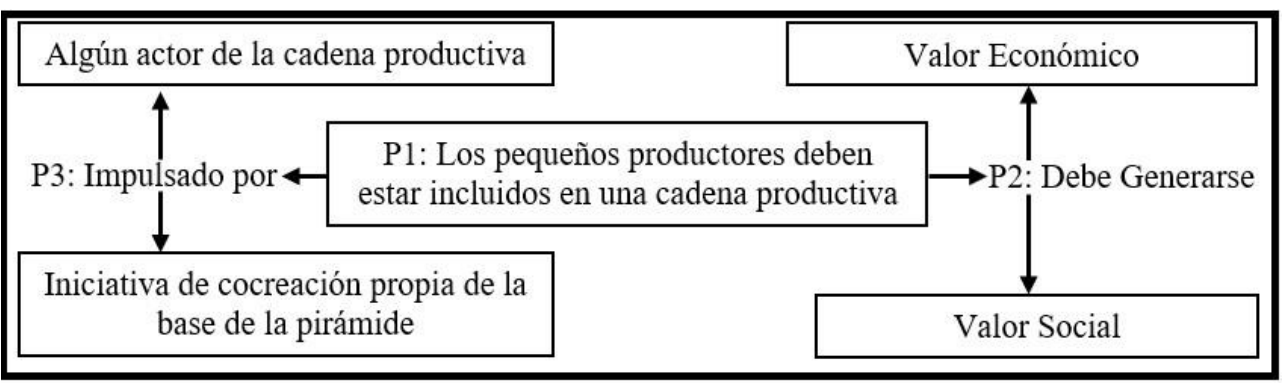

Fuente: elaboración propia 


\section{Metodología}

La presente investigación se desarrolló bajo la metodología del estudio de casos. Esta metodología es aplicable cuando hay muchas más variables que datos observacionales en un fenómeno que puede ser analizado en su contexto de vida real, sobre todo cuando los límites entre el fenómeno y el contexto no son claramente evidentes. Este es el caso de las cooperativas bananeras del Magdalena (Colombia) que se estudia en esta investigación.

En el estudio de casos los resultados son validados con las evidencias de las múltiples fuentes que convergen de forma triangular, cuyos datos recolectados son guiados por las proposiciones teóricas formuladas anteriormente a partir de la revisión de literatura (Yin, 1989).

Por lo anterior, el diseño del estudio de caso de esta investigación se realizó siguiendo la metodología planteada por Yin (1989) que incluye cinco pasos importantes: 1. Formulación de una pregunta de investigación, 2. Establecimiento de proposiciones 3. Definición de la unidad de análisis, 4. Análisis de los datos con las proposiciones formuladas 5. Interpretación de los resultados.

Los pasos uno (1) y dos (2) han sido explicados en los apartados anteriores. Por lo que a continuación se desarrollará la implementación del resto de las etapas:

\section{Descripción de la unidad de análisis:}

La unidad de análisis seleccionada está compuesta por la totalidad de las cooperativas bananeras del Departamento del Magdalena (Colombia) (6 cooperativas), conformando un estudio de casos múltiples, lo que permite que una teoría que ha sido analizada y plasmada como una plantilla teórica, pueda ser comparada con los datos de un caso y apoyada por las repeticiones de los resultados de los múltiples casos. El número de casos estudiados está en consonancia con lo indicado por Eisenhardt (1989), que muestra como rango aceptable para el estudio un número de cuatro a diez casos, argumentando que si el número es inferior a cuatro, es difícil generar teoría y pueden surgir problemas empíricos.

Las cooperativas bananeras del Magdalena reúnen un total de 335 asociados y 35 empleados, como se muestra en la tabla 2 y se encuentran ubicadas en Santa Marta y la Zona Bananera del departamento del Magdalena (Colombia). Se caracterizan por agrupar a pequeños productores de banano de exportación certificado por Global Gap y Comercio Justo.

Tabla. 2. Unidad de análisis

\begin{tabular}{llll}
\hline COOPERATIVA & Ubicación & No. de Socios & No. de Empleados \\
\hline A & Río Frío & 31 & 5 \\
B & Santa Marta & 36 & 5 \\
C & Río Frío & 53 & 6 \\
D & Guacamayal & 62 & 8 \\
E & Orihueca & 78 & 8 \\
F & Orihueca & 75 & 9 \\
\hline Total & & $\mathbf{3 3 5}$ & $\mathbf{3 5}$ \\
\hline
\end{tabular}

Fuente: elaboración propia 


\section{Recolección de información:}

El proceso de recolección de información se hizo en el período de Febrero 2016-Mayo 2017, en el departamento del Magdalena, Colombia. Se realizaron visitas a las cooperativas y fincas de los productores, en donde se desarrollaron las entrevistas, encuestas y observación directa del proceso de producción y embarque del banano.

Para la recolección de los datos se utilizaron diferentes fuentes de información (tabla 3):

- Entrevistas: Se realizaron 46 entrevistas en las cooperativas, correspondientes a 32 socios que forman parte de los órganos directivos, 11 empleados que ocupan puestos de responsabilidad en la cooperativa y 3 empleados (supervisores) de comercializadoras ${ }^{6}$. El cuestionario semiestructurado utilizado para la recolección de información fue diseñado una vez se hizo la revisión de la literatura y se estableció el modelo teórico de las características que cumplen los agronegocios inclusivos con cooperativas. El enfoque de las preguntas realizadas a los socios directivos y a los empleados de las cooperativas estaba relacionado con el origen, funcionamiento de la cooperativa y los cambios logrados por los pequeños productores después de su vinculación.

Por otro lado, las preguntas realizadas a los supervisores tenían como objetivo conocer las características de la comercializadora, su rol y la relación con la cooperativa.

Encuestas: Se realizaron encuestas a 237 pequeños productores (socios) pertenecientes a las seis cooperativas existentes, a través de un cuestionario estructurado con preguntas relacionadas con la producción, rentabilidad del negocio, estrategias de marketing y comercialización, manejo de los recursos físicos y del talento humano, entre otras.

Tabla. 3. Resumen de la recolección de la información

\begin{tabular}{llll}
\hline \multirow{2}{*}{ EMPRESA } & \multicolumn{2}{l}{$\begin{array}{l}\text { No. de } \\
\text { Encuestas }\end{array}$} & No. de Entrevistas \\
\cline { 2 - 4 } & Socios & $\begin{array}{l}\text { Socios } \\
\text { Directivos }\end{array}$ & Empleados \\
\hline COOPERATIVA A & 20 & 4 & 1 \\
COOPERATIVA B & 22 & 4 & 1 \\
COOPERATIVA C & 42 & 4 & 2 \\
COOPERATIVA D & 45 & 6 & 2 \\
COOPERATIVA E & 56 & 7 & 2 \\
COOPERATIVA F & 52 & 7 & 3
\end{tabular}

6 El artículo 40 del decreto 380 de 2012, expedido por el Ministerio de Hacienda y Crédito Público (2012) define las comercializadoras como aquellas personas jurídicas que tienen por objeto social principal la comercialización y venta de productos colombianos al exterior, adquiridos en el mercado interno o fabricados por productores socios de las mismas. 


\begin{tabular}{llll} 
COMERCIALIZADORA 1 & & & 1 \\
COMERCIALIZADORA 2 & & & 1 \\
COMERCIALIZADORA 3 & & & 1 \\
\hline Total & $\mathbf{2 3 7}$ & $\mathbf{3 2}$ & $\mathbf{1 4}$ \\
\hline
\end{tabular}

Fuente: elaboración propia

Así mismo, se utilizó información secundaria como informes técnicos de Comercio Justo, de cadenas productivas y comercialización de la FAO, páginas web, revistas, entre otros.

\section{Análisis de la información:}

Las entrevistas fueron transcritas en formato Word y las encuestas se tabularon en Excel.

Para el análisis de las entrevistas se utilizó el software de análisis cualitativo de datos, Atlas.ti v 7.0. que permitió definir las categorías y relacionarlas con los resultados encontrados en la literatura.

Las entrevistas con los socios directivos y los trabajadores de las cooperativas permitieron conocer el rol del productor en la cadena productiva, los inicios y desarrollos de la organización cooperativa a la que pertenecen y los cambios más significativos en la calidad de vida del pequeño productor.

Finalmente, las entrevistas a los miembros de la comercializadora dieron a conocer información de las relaciones comercializadora - cooperativa - pequeño productor y el proceso de venta al exterior del banano.

Por otro lado, las encuestas del pequeño productor asociado a las cooperativas, ayudaron a diagnosticar sus condiciones actuales a nivel formativo, organizacional y de producción; permitiendo obtener una caracterización actualizada de la unidad de análisis.

Todos esos aspectos analizados con las entrevistas y las encuestas, fueron corroboradas con la observación directa y la información secundaria, permitiendo la triangulación de todas las fuentes recolectadas, encontrando repeticiones comportamentales del fenómeno en las diferentes cooperativas y la generalización analítica que valida la teoría.

\section{Resultados y discusión}

Al contrastar los datos analizados de las cooperativas bananeras del Magdalena con las proposiciones teóricas de las características de los negocios inclusivos que se desarrollan en cooperativas agrarias, se pueden evidenciar los siguientes resultados y su validación o rechazo de las proposiciones establecidas. 


\subsection{Los pequeños productores incluidos en la cadena de valor del Banano de Colombia que hacen parte de la base de la pirámide agraria}

Basado en el análisis de la información primaria recolectada se concluye que la cadena productiva del banano del departamento del Magdalena que incluye al pequeño productor, tiene los siguientes actores involucrados:

Figura. 2. Cadena productiva del banano que incluye al pequeño productor.

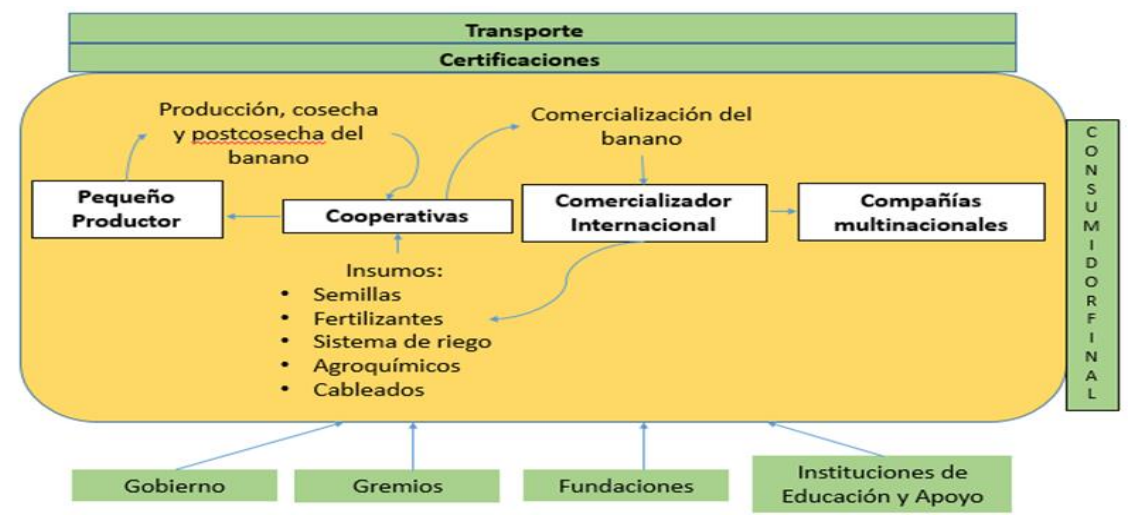

Fuente: elaboración propia a partir del análisis de la información

Los productores, según los resultados de las encuestas, se encargan de desarrollar las labores de las etapas de pre-cosecha, cosecha y post-cosecha de la fruta. Pueden ser, por un lado, grandes y pequeños agricultores que trabajan de forma independiente; y por otro, pequeños agricultores asociados a través de cooperativas. Los pequeños agricultores (independientes o asociados) que están incluidos en el negocio del banano de exportación en el Magdalena se pueden considerar como la base de la pirámide de la cadena de valor. Estos productores cuentan con una extensión de tierra que va de 0,35 a 11,87 hectáreas, siendo el promedio de 3,15 hectáreas. Los trabajadores de las fincas tienen un nivel educativo bajo: un $69 \%$ de ellos no cuentan con educación formal, el $17 \%$ ha finalizado la educación básica primaria, el 12\% bachiller académico y solo el $1 \%$ posee estudios técnicos u otros estudios.

Los pequeños productores entrevistados definen las cooperativas como instituciones intermediarias entre ellos y las empresas comercializadoras, que facilitan el desarrollo de las actividades de ambas partes: a los pequeños agricultores porque garantizan la venta de su producto a mercados a los que de forma independiente no pueden llegar y a las comercializadoras porque el contar con un solo proveedor que les proporcione la cantidad de banano requerido (Jimeno, 2017) disminuye los costos de transacción de sus operaciones.

Los supervisores de las comercializadoras afirman que la venta del banano del Magdalena se realiza a nivel internacional (Centroamérica y el Caribe, Norte América y Europa) a través de cadenas de exportación especializadas. 
Por otro lado, los directivos y técnicos entrevistados indicaron que el sistema de transporte de la cadena de valor incluye vehículos pesados (suministrados por las cooperativas) y traslado en buque hacia los puertos de destino, siendo Estados Unidos el principal destino (Ministerio de Tecnologías de la Información y las Comunicaciones, 2018 y EUROSTAT, 2018)

Según los pequeños productores y los supervisores de las comercializadoras que participaron en el estudio, dentro de las exigencias de exportación solicitadas por el cliente, se encuentra la certificación por Global Gap como requisito mínimo y la de Comercio Justo como certificación opcional, por lo que todas las cooperativas tienen ambas certificaciones y una de ellas está en proceso de evaluación de la certificación de banano orgánico.

Para la obtención de las certificaciones, los pequeños productores afirman que ICONTEC es el ente certificador autorizado para el Global Gap y Fairtrade Labelling Organizations International (FLO) para el comercio justo. Además, reconocen que existen otras organizaciones que han ayudado al proceso de formación y apoyo del pequeño productor, entre las que se encuentran dos asociaciones (AUGURA y ASBAMA), dos fundaciones (Fundauniban y Fundación Banasan) y dos instituciones de educación (Servicio Nacional de Aprendizaje -SENA- y Universidad del Magdalena).

Estos resultados están en línea con los presentados por Ostertag et al. (2014) que indican en su estudio que la cadena de valor del banano en Colombia incluye: proveedores de insumos (cajas, sellos, plásticos, fertilizantes, fumigación aérea), transportadores fluviales y marítimos, puertos, terminales de contenedores, asociaciones comerciales, sindicatos, empresas bananeras, productores bananeros grandes y pequeños, pequeños productores agrupados en cooperativas, exportadores, importadores, minoristas (principalmente supermercados en la Unión Europea y los EE. UU.), fundaciones del sector privado y agencias que brindan servicios de apoyo técnico y social.

Otros estudios, liderados por el SENA - Servicio Nacional de Aprendizaje (2013) evidencian una cadena que incluye actores y procesos similares a los estudiados, abarcando toda Colombia y diferentes tipos de cultivos.

\subsection{Generación de valor económico y valor Social}

Para el análisis y presentación de los resultados en este apartado se ha diferenciado entre la generación de valor económico y la generación de valor social.

Generación de valor económico:

Los socios directivos y los supervisores de la comercializadora opinan que los productores y mayoristas son los actores a los que les corresponde el menor porcentaje del valor económico de la cadena productiva del banano de exportación a Europa; mientras que los importadores y minoristas reciben las mayores ganancias a pesar de los incrementos en los precios de compra del banano de exportación en los últimos años, opinión que reafirma los datos de la FAO (2018) (ver tabla 4). 
Además, los socios directivos afirmaron en sus entrevistas que los precios del banano en el mercado internacional están influenciados por los precios del combustible, que afecta el costo de transporte de la fruta.

Sin embargo, la certificación de los pequeños productores con Comercio Justo ha garantizado el pago regulado de sueldos de los trabajadores, el cumplimiento de las condiciones mínimas de trabajo enfocadas a la salud e higiene industrial y la no contratación de mano de obra infantil o forzosa, como mostraron las encuestas a los pequeños productores en la sección del manejo del talento humano.

De igual forma, el hecho de que la mayoría de los pequeños productores tengan la certificación de Comercio Justo garantiza que los trabajadores, productores, transportadores y consumidores obtengan mayores beneficios y trabajen de acuerdo a las regulaciones legales.

Así mismo, los socios directivos confirmaron en las entrevistas realizadas, que el pequeño productor recibe 1 USD adicional por cada caja vendida certificada con Comercio Justo. Esto permite que el porcentaje del valor económico correspondiente a este eslabón se duplique en ambos mercados (ver tabla 4).

Estos resultados son similares a los que han publicado sobre el sector algunas asociaciones como ASBAMA $(2016,2017)$ que muestran la recuperación y prosperidad de todos los actores de la cadena productiva del banano en estos últimos dos años, teniendo en cuenta el aumento en la cotización del dólar y la dinámica del mercado. De igual forma, el estudio realizado por Ostertag et al. (2014) sobre los impactos del Comercio Justo muestra cómo los pequeños productores han sido favorecidos por el cultivo del banano y su venta bajo la certificación de comercio justo. 
Tabla 4. Valor económico por eslabón de la cadena productiva del banano

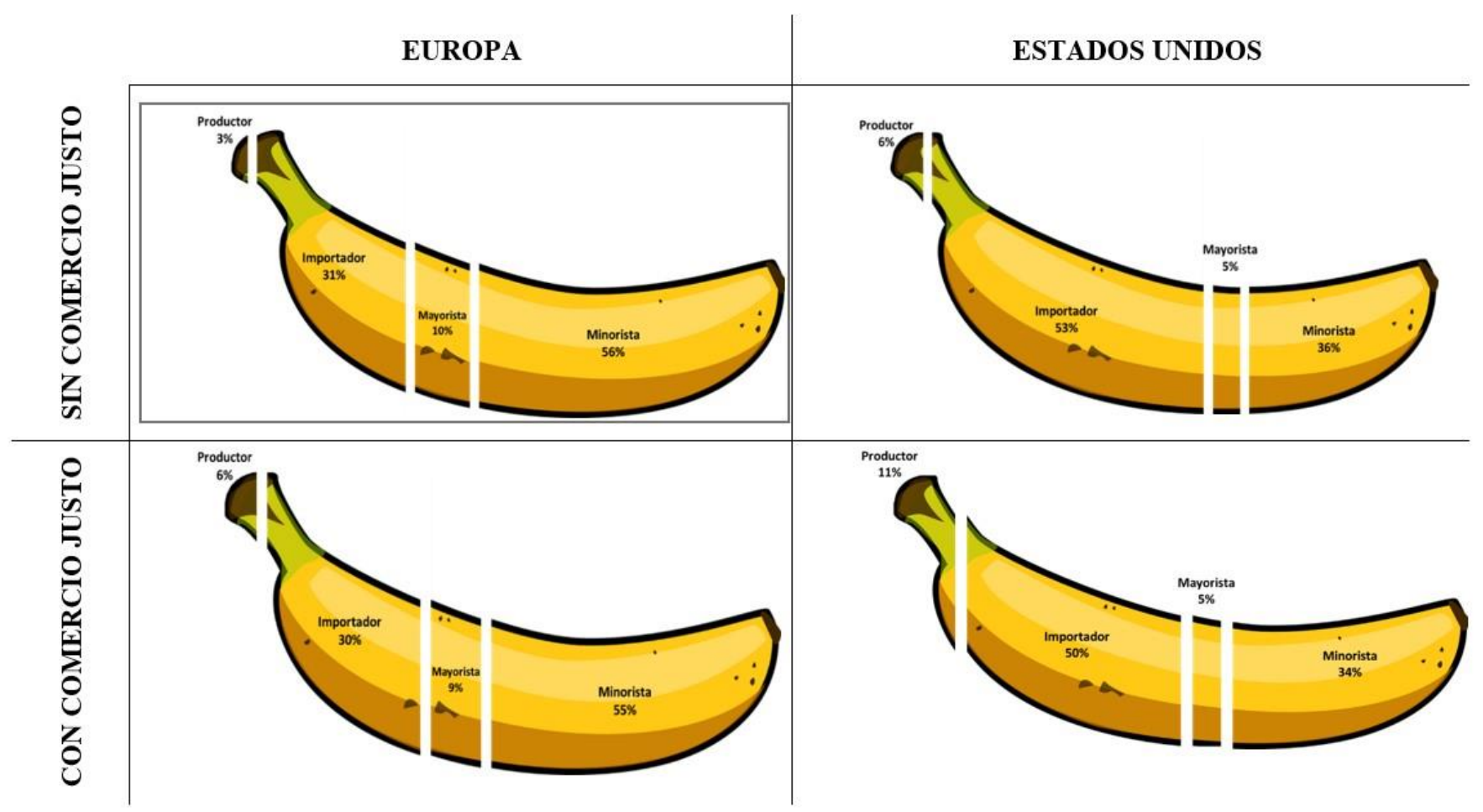

Fuente: Elaboración propia a partir de datos de la Organización de las Naciones Unidas para la Agricultura y el Desarrollo (FAO), 2018 
- Generación de valor social:

Según el análisis de la información primaria, los pequeños productores han tenido cambios positivos con el desarrollo del negocio inclusivo y con los beneficios obtenidos por la certificación de Comercio Justo, ya que además de cumplir con las condiciones establecidas de tener un precio de compra estable y garantía de la compra de su producción, obtienen una prima adicional de un 1 USD por caja vendida; lo que les ha permitido recibir mayores ingresos que han ayudado a mejorar sus condiciones de vida y la de sus familiares.

En las entrevistas analizadas, los productores manifiestan que la prima adicional recibida es utilizada para la ejecución de proyectos en vivienda, educación, infraestructura, préstamos a nivel de los productores y mejora de las fincas. En el caso de las cooperativas, este valor adicional ha sido utilizado para la realización de capacitaciones, apoyo para el mantenimiento de la norma de Comercio Justo y algunas inversiones en bienestar social de la comunidad.

Debido a las mejoras económicas y sociales de los participantes en el negocio inclusivo, ha aumentado la confianza entre los pequeños productores y las cooperativas. Los entrevistados expresaron los fuertes lazos de hermandad y fraternidad que existen entre ellos, que generan lo que en la literatura se denomina capital social y que les han permitido afrontar las dificultades y mejorar sus condiciones de vida.

Así mismo, los pequeños productores reconocen la gran ayuda que han tenido, no solo a nivel económico, sino también a nivel social, al formar parte activa de una cadena productiva y generar ingresos a través de su propio emprendimiento como agricultores.

Por otro lado, los pequeños productores indicaron que las organizaciones ASBAMA, AUGURA y las Fundaciones de Banasan y Fundaunibán han desarrollado diferentes proyectos a lo largo de los años, que tienen como objetivo ayudar a las cooperativas, a los pequeños productores y a la comunidad en general. Dentro de los proyectos realizados se encuentran capacitaciones técnicas y administrativas, asistencia técnica, formación en diferentes áreas, jornadas de limpieza, de salud, convenios relacionados para tratar el cambio climático y velar por la regulación del agua, entre otros. Se puede concluir por ello que todos los actores trabajan de forma colaborativa y en pro del beneficio social de los pequeños productores.

Estos resultados se encuentran alineados con los mostrados por el informe de impacto de Comercio Justo (Fairtrade Internacional, 2013), realizado en las cooperativas bananeras del Magdalena; en donde mencionan que en promedio, el 35\% de la prima Comercio Justo se invierte en la productividad de la finca y en la reducción de los costos de producción del banano,; el 15\% se gasta para cubrir los costos de administración, el 12\% se usa para pagar el mantenimiento de la norma Comercio Justo y el $10 \%$ restante se invierte en el bienestar social en la comunidad.

Así mismo, en diferentes estudios con cooperativas agrarias se demuestra que la inclusión en una cadena productiva mejora las condiciones de vida de los pequeños productores (Ickis et al., 2009; Licandro, 2013). De igual 
forma, autores como Mellor (1999) ha demostrado que el crecimiento agrícola produce una reducción de la pobreza en países con alto índice de pobreza.

\subsection{Inicio y consolidación de la cooperativa motivada por algún actor de la cadena productiva y/o por iniciativa de cocreación propia de la base de la pirámide}

Al hacer un análisis de las entrevistas realizadas con relación al origen de las cooperativas se pudo identificar que la comercializadora, las exigencias del Comercio Justo y la cocreación de la base de la pirámide (BoP) son los tres factores que han impulsado el nacimiento del negocio inclusivo (Ver tabla 5).

Tabla. 5. Resumen del origen y factor que ha impulsado la creación de la cooperativa

\section{Cooperativa Año de Fundación Factor que da inicio a la cooperativa}

\begin{tabular}{|c|c|c|}
\hline A & 1987 & Comercializadora \\
\hline B & 2007 & Exigencias del Comercio Justo \\
\hline $\mathrm{C}$ & 1999 & Coocreación de la BoP \\
\hline $\mathrm{D}$ & 1997 & Coocreación de la BoP \\
\hline $\mathrm{E}$ & 1979 & $\begin{array}{l}\text { Inicialmente nace como Asociación } \\
\text { producto de la Coocreación de la BoP y } \\
\text { luego se transforma a cooperativa por } \\
\text { exigencias del Comercio Justo }\end{array}$ \\
\hline $\mathrm{F}$ & 1998 & $\begin{array}{l}\text { Inicialmente nace como empresa privada } \\
\text { y luego se transforma a cooperativa por } \\
\text { exigencias del Comercio Justo }\end{array}$ \\
\hline
\end{tabular}

Fuente: Elaboración propia

La cooperativa A tiene su origen motivado en la solicitud de la comercializadora del momento, teniendo en cuenta que agilizaba la gestión de compra a una sola cooperativa que agrupara a los pequeños productores, más que la compra individual a cada productor.

Por su parte la cooperativa B nace directamente de la necesidad de cumplir con las exigencias de los mercados internacionales del Global Gap y Comercio Justo, como manifestaron los entrevistados de dicha cooperativa. Los pequeños productores analizaron los requisitos de la certificación y los beneficios que podrían obtener al lograrla, decidiendo asociarse para unir fuerzas, ser incluidos en la cadena productiva y mejorar a través del negocio inclusivo su calidad de vida.

Las cooperativas $\mathrm{C}$ y $\mathrm{D}$ surgieron por el interés de los mismos pequeños productores de trabajar unidos, generar apoyo económico y moral y lograr un poder de negociación frente a las comercializadoras. Adicional a esto, las condiciones ambientales de la zona y la gran demanda en el mercado internacional hicieron 
evidente la oportunidad de asociarse y emprender este nuevo proyecto como cooperativas. Además, analizaron que los recursos obtenidos por la venta de fruta en el mercado exterior a través de las comercializadoras eran superiores a los pagados por el mercado local, siendo entonces el cultivo de exportación uno de sus principales objetivos.

La cooperativa $\mathrm{E}$ por su parte, si bien surge por iniciativa de los pequeños productores y la oportunidad presentada en el mercado por la comercializadora de comprar cajas de banano a pequeños agricultores, tiene como particularidad que nace como asociación y no como cooperativa. Sin embargo, las mismas exigencias del mercado y la oportunidad de certificarse con Comercio Justo marcaron un camino de recuperación financiera en estos productores, siendo el primer paso su transformación de asociación a cooperativa, cumpliendo uno de los requisitos exigidos por dicha certificación.

La cooperativa $\mathrm{F}$ en cambio, surge de la transformación de una empresa privada a cooperativa. La empresa privada ayudaba a la mejora productiva de las fincas, ofreciendo asistencia técnica y préstamos, lo que originó una excelente relación entre propietarios y pequeños productores. Sin embargo, los pequeños productores al conocer los beneficios que estaba otorgando el Comercio Justo en las otras cooperativas y la nueva oportunidad de mercado, propusieron la compra de la empresa y el traspaso a cooperativa con el fin de cumplir las exigencias de esta certificación, siendo entonces 46 pequeños productores los que inician el proceso de traslado legal a cooperativa.

Teniendo en cuenta lo anterior, se puede afirmar que el nacimiento de tres cooperativas (E, F y B) se da por las exigencias planteadas por las regulaciones del mercado internacional y no por impulso de los propios productores o de algún actor de la cadena.

Con relación al respaldo del desarrollo del negocio inclusivo, se identificó a las comercializadoras como el "actor clave de la cadena productiva" para su desarrollo, ya que además de ser las intermediarias entre el cliente internacional y las cooperativas agrarias que asocian a los pequeños productores, velan por la generación de valor compartido para todos, además de brindar asistencias técnicas, capacitaciones al pequeño productor y apoyo en proyectos comunitarios, entre otros.

Por otro lado, aparece un nuevo actor que ha impulsado el desarrollo del negocio inclusivo y es la responsabilidad ética del consumidor final, que está dispuesto a pagar un valor adicional por velar que se genere el comercio justo en la cadena productiva. Este valor adicional, como se ha mostrado en los apartados anteriores ha ayudado a la mejora del pequeño productor y ha permitido garantizar el cumplimiento de condiciones mínimas legales para todos los miembros de la cadena productiva.

Los resultados encontrados validan la proposición teórica de la existencia de actores que motivan la generación y desarrollo del negocio inclusivo, pero incluyen un nuevo factor que son las exigencias normativas y legales del mercado internacional, como es el Comercio Justo.

Estos hallazgos van en concordancia con diversos trabajos realizados sobre negocios inclusivos y casos de estudio del agro (Márquez et al., 2010; Van Haeringen y de Jongh, 2010; Licandro, 2013; Bustamante y Muñoz, 2017). 


\subsection{Replicable y/o escalable a lo largo del tiempo}

Al hacer un análisis del nacimiento y evolución de las cooperativas, se encontró que las dos más antiguas: A (creada en 1987 por 17 socios) y E (creada en 1979 por 26 socios) nacen como respuesta a los problemas de desabastecimiento del banano, consecuencia de la alta demanda del mercado.

El impacto positivo de las cooperativas en el negocio se hizo evidente en la década de 1990; los pequeños productores notaron los beneficios de incorporarse al modelo de negocio cooperativo (facilidad en las transacciones, acceso al mercado internacional, apoyo mutuo) y para ese período (década de 1990) las organizaciones pasaron de tener 43 asociados a contar con 420 socios en total.

Adicionalmente, a finales de los 90's nacen otras dos cooperativas: C y D y una empresa privada que se convierte posteriormente en F.

Esta cercanía del año de origen de tres (C, D y F) de las seis cooperativas, muestra que en el período 1990-2000 el banano estuvo beneficiado por diferentes acontecimientos externos. Por un lado, se firman convenios entre Europa y Colombia, que incluían una garantía desde el año de 1995 de comprar 2,35 millones de toneladas de banano al año. Con Estados Unidos se produjo un aumento significativo de las exportaciones y se generó la primera exportación de una asociación de bananeros de forma directa, garantizándole una participación equivalente al 0,5 por ciento de la cuota colombiana, es decir 1.500 .000 cajas de banano (Nullvalue, 2011). Y por último, en 1997 se inicia todo el proceso de EUREPGAP, que después se convertirá en Global Gap junto con el nacimiento de FLO y las certificaciones de Comercio Justo, que mostraron desde sus inicios una buena perspectiva para el mercado de exportación del banano, especialmente para el pequeño productor.

La última cooperativa que nace en el 2007 (B) expresó que sus inicios se hicieron motivados por las condiciones favorables que estaba brindando el banano de exportación, principalmente por los negocios inclusivos que se estaban generando y los beneficios que estaban obteniendo las otras cooperativas con el comercio justo.

Por lo anterior y como se observa en la tabla 6, a través del tiempo han aparecido nuevas cooperativas y ha aumentado significativamente el número de asociados de cada una de ellas, siendo $\mathrm{E}$ y $\mathrm{F}$ las de mayor variación desde su constitución. Otro caso atípico lo mostró A, al pasar de 44 a 31 asociados del año 2013 a 2017; sin embargo, al compararla con el número inicial de socios ha mostrado un incremento de 14 miembros. La cooperativa A actualmente está en un proceso de recuperación organizativa, debido a incidentes presentados por la administración anterior, situación que se ve reflejada en la disminución de socios en los últimos cuatro años. 
Tabla. 6. Replicación del modelo de negocio inclusivo a través de cooperativas

\begin{tabular}{|c|c|c|c|c|c|c|}
\hline \multirow{2}{*}{ COOPERATIVA } & \multirow{2}{*}{ Año de Fundación } & \multicolumn{3}{|c|}{ No. De Socios } & \multicolumn{2}{|c|}{$\begin{array}{l}\text { Variación en el No } \\
\text { De Socios }\end{array}$} \\
\hline & & Inicial & 2013 & 2017 & $\begin{array}{l}(2017- \\
2013)\end{array}$ & $\begin{array}{l}(2017 \\
\text { Inicial })\end{array}$ \\
\hline A & 1987 & 17 & 44 & 31 & -13 & 14 \\
\hline B & 2007 & 26 & 33 & 36 & 3 & 10 \\
\hline $\mathrm{C}$ & 1999 & 31 & 42 & 53 & 11 & 22 \\
\hline $\mathrm{D}$ & 1997 & 37 & 40 & 62 & 22 & 25 \\
\hline $\mathrm{E}$ & 1979 & 26 & 80 & 78 & -2 & 52 \\
\hline $\mathrm{F}$ & 1998 & 36 & 59 & 75 & 16 & 39 \\
\hline Total & & 173 & 298 & 335 & & \\
\hline
\end{tabular}

Fuente: Elaboración propia

Con lo anterior, se puede observar que el modelo iniciado por las cooperativas A y E, ha sido replicado y/o escalado en el tiempo por las cuatro (4) cooperativas que han ido conformándose desde finales de los 90’s hasta el 2007, motivado por los beneficios del trabajo conjunto y las exigencias del comercio justo.

Al analizar casos similares, se encontró que Dole en Perú, muestra un comportamiento parecido con productores orgánicos de exportación, ya que esta multinacional ha invertido en los agricultores asociados para reducir el riesgo de su negocio y garantizar el cumplimiento de la demanda. En contraprestación, los pequeños productores reciben beneficios, garantizan las ventas de su producción y mejoran sus condiciones económicas y de calidad de vida.

Otros casos similares son los de CISA-Exportadora (Nicaragua) y CIGRAH (Honduras), cuyo producto de exportación es el café certificado, con el sello internacional Utz Certified para la producción social y ambiental sostenible, ayudando a la venta de café a un mejor precio. Ambos casos fueron analizados por Van Haeringen y de Jongh ${ }^{(2010)}$.

Márquez et al., (2010) muestran casos de agronegocios que se han desarrollado con cooperativas como Apaeb en Brasil y recolectores de Bío-Bío de Chile, que han sido exitosos e inclusivos. Finalmente, en Colombia, (SNV - Servicio Holandés de Cooperación al Desarrollo y CECODES - Consejo Empresarial Colombiano para el Desarrollo, 2008) han reconocido como negocios inclusivos relacionados con el agro a Indupalma, Juan Valdés y Compañía Nacional de Chocolates, teniendo en cuenta la vinculación del pequeño productor en los diferentes actores de la cadena. 


\section{Conclusiones}

Las cooperativas agrarias son consideradas como un sistema organizacional propicio para el desarrollo de un negocio inclusivo teniendo en cuenta sus principios, la estructura orgánica que las caracteriza y las leyes que las rigen. Sin embargo, hay unas características definidas por los negocios inclusivos, que fueron identificadas en esta investigación y que permiten determinar si una cooperativa agraria está llevando a cabo o no un negocio de inclusión económica y social de la base de la pirámide.

La primera de las características identificadas es la vinculación del pequeño productor en alguna actividad de la cadena de valor del producto. En el caso de las cooperativas bananeras del Magdalena, se estableció que la cadena de valor está conformada por los siguientes actores: i) Productores asociados en cooperativas; ii) Comercializadoras; iii) Transportadores; iv) Importadores (Compañías Multinacionales). Además, se da la participación de otras organizaciones o instituciones que intervienen con los diferentes actores, como los entes certificadores, las fundaciones y asociaciones y las instituciones de educación y apoyo.

Los pequeños productores son considerados la base de la pirámide del sistema agrario, están en el inicio de la cadena de valor y son los encargados de proveer la fruta que llega hasta el consumidor final. Así mismo, tienen relación directa con casi todos los actores de la cadena exceptuando el cliente, siendo la comercializadora la encargada de establecer ese contacto.

La segunda característica encontrada en la investigación es la generación de valor económico y valor social con el negocio. Dicho valor económico viene establecido por la rentabilidad de la empresa, el cumplimiento de condiciones favorables y legales en cada uno de los actores de la cadena, el precio justo pagado, las relaciones comerciales a largo plazo y los pagos oportunos. El valor social por su parte, está enfocado a la mejora de las condiciones de vida de los pequeños productores, la construcción del capital social entre ellos, el desarrollo de competencias empresariales y el acceso a la información.

Con relación a la tercera característica, se demuestra que los negocios inclusivos en las cooperativas agrarias pueden ser impulsados por algún actor de la cadena de valor o por iniciativa propia de los pequeños productores. Sin embargo, se evidenció un nuevo factor que hace referencia a las exigencias normativas y legales del mercado internacional, en donde los auditores de la misma velan por la existencia de la inclusión y el valor compartido.

La última característica establecida hace referencia a la replicabilidad y/o escalabilidad del modelo de negocio inclusivo y está relacionada con la creación de nuevas cooperativas agrarias del mismo producto o productos diferentes o con el aumento de números de productores que hacen parte del negocio.

Dentro de las limitaciones presentadas en esta investigación se puede mencionar el número de casos estudiados (6 cooperativas bananeras correspondientes al censo total de este tipo de empresas en la zona de estudio). Aunque este número de unidades de análisis podría considerarse pequeño para un estudio representativo; con los datos recolectados, la triangulación de la información y la comparación con otras investigaciones, se pudieron establecer generalizaciones para cooperativas 
agrarias con características similares. En términos académicos las proposiciones validadas en este artículo proponen un modelo de cooperativa agrícola con inclusión social, el cual puede ser replicado en países con altos índices de desigualdad y ser utilizado como una estrategia de erradicación de la pobreza.

Como futuras investigaciones se sugiere realizar un comparativo entre cooperativas de grandes productores y cooperativas con pequeños productores (base de la pirámide), con el fin de establecer diferencias conceptuales de modelos de negocios. Así mismo, se sugiere como segunda fase de esta investigación, replicar la investigación con un mayor número de cooperativas similares de otros productos, para validar con otra unidad de análisis las proposiciones aquí planteadas.

\section{Referencias bibliográficas}

ASBAMA. (2016) Informe de Gestión 2015. Santa Marta.

ASBAMA. (2017) Informe de Gestión 2016. Santa Marta.

Betancourt Latorre, A. V. (2014) Propuesta metodólogica para la creación de negocios inclusivos en el LANI-EAFIT Social. Universidad EAFIT.

Bustamante, C. E., y Muñoz, Y. (2017) Propuesta de negocio inclusivo: hacia un modelo de inclusión social de sacha inchi en el Bajo Cauca antioqueño. Equidad y Desarrollo, $\mathrm{N}^{\mathrm{o}}$ 27, pp. $125-147$.

CONFECOOP - Confederación de Cooperativas de Colombia. (2009) Las Cooperativas de Trabajo Asociado en Colombia. In Observatorio cooperativo (Vol. 11).

CONFECOOP - Confederación de Cooperativas de Colombia. (2017) Cooperativas: Empresas para la gestión social y económica. Bogotá D.C.

Congreso de Colombia. (1998) Ley 454 de 1998.

Coraggio, J. L. (2004) Una alternativa socioeconómica necesaria: la economía social. Deba Tes Fundamentales, Buenos Aires, Altamira UNGS.

Corporación Financiera Internacional. (2012) Policy Note on the Business Environment for Inclusive Business Models. Retrieved from http://www.ifc.org

Eisenhardt, K. M. (1989) Agency Theory: An Assessment and Review. Academy of Management Review, $\mathrm{N}^{\circ} \quad 14, \quad$ Vol. $1, \quad$ pp. 57-74. https://doi.org/10.5465/amr.1989.4279003.

EUROSTAT. (2018) EU trade since 1988 by CN8. Retrieved July 19, 2018, from http://appsso.eurostat.ec.europa.eu/nui/show.do?query=BOOKMARK_DS-

016890_QID_-6F9E51AC_UID_-

3F171EB0\&layout=PERIOD,L,X,0;REPORTER,L,Y,0;PARTNER,L,Z,0;PRODUCT, C,Z,1;FLOW,L,Z,2;INDICATORS,C,Z,3;\&zSelection=DS-016890FLOW,1;DS016890PARTNER,EU28_EXTRA;DS-0168

Fairtrade Internacional. (2013) Informe Anual 2012-2013. Germany.

FAO - Organización de las Naciones Unidas para la Alimentación y la Agricultura. (2018) Trade and Markets. Retrieved June 20, 2018, from http://www.fao.org/economic/est/estcommodities/bananas/bananafacts/en/\#.WyArjEgvzIX

FAO - Organización de las Naciones Unidas para la Alimentación y la Agricutura. (2012) FAO y CEPAL celebran Año Internacional de las Cooperativas junto a 250 agricultores chilenos y líderes mutualistas de América Latina. Retrieved from http://www.fao.org/americas/noticias/ver/es/c/229694/ 
Fisac Garcia, R., Moreno Romero, A. M., Mataix Aldeanueva, C., y Palacios Fernández, M. (2011) La empresa social: revisión de conceptos y modelo para el análisis organizativo. Revista Española Del Tercer Sector, pp. 41-66.

Fundación AVINA. (2008) Liderazgo para el Desarrollo Sostenible en América Latina.

Gatica, J. L., Muñoz, L., Valenzuela, K., Espinoza, M., Zapata, S., y De La Parra, R. (2012) Análisis de test de parche en el Hospital Clínico de la Universidad de Chile. Período 2000-2009. Piel, No 27, Vol. 6, pp. 300-303. https://doi.org/10.1016/j.piel.2012.01.016

Golja, T., y Požega, S. (2012) Inclusive business-what it is all about? Managing inclusive companies. International Review of Management and Marketing, $\mathrm{N}^{\mathrm{o}} 2$, Vol. 1, pp. 22 42.

Gradl, C., y Knobloch, C. (2010) Inclusive Business Guide How to Develop Business and Fight Poverty Publication information. Retrieved from www.endeva.org

Hahn, R. (2012) Inclusive business, human rights and the dignity of the poor: a glance beyond economic impacts of adapted business models. Business Ethics: A European Review, № 21, Vol. 1, pp. 47-63.

Ickis, J. C., Leguizamón, F. A., Metzger, M., y Flores, J. (2009) La agroindustria: campo fértil para los negocios inclusivos. Academia. Revista Latinoamericana de Administración, $\mathrm{N}^{\circ} 43$.

Ishikawa, A., y Strandberg, L. (2009) Negocios inclusivos: creando valor para las empresas y para la población de bajos ingresos. Cuadernos de La Cátedra La Caixa de Responsabilidad Social de La Empresa y Gobierno Corporativo. España: Business School, Universidad de Navarra.

Jimeno, D. (2017) Entrevista a productor asociado. Zona Bananera del Magdalena, Colombia.

Kandachar, P., y Halme, M. (2008) Sustainability Challenges and Solutions at the Base of the Pyramid: Business. Technology and the Poor (Greenleaf, London).

Karnani, A. (2007) The mirage of marketing to the bottom of the pyramid: How the private sector can help alleviate poverty. California Management Review, $\mathrm{N}^{\mathbf{0}} 49$, Vol. 4, pp. 90-111.

Licandro, O. D. (2013) Modelos para el análisis de los negocios inclusivos: construcción mediante el estudio de casos. Telos: Revista de Estudios Interdisciplinarios En Ciencias Sociales, $\mathrm{N}^{\circ} 15$, Vol. 1, pp. 32-48.

London, T., y Hart, S. L. (2010) Next generation business strategies for the base of the pyramid: New approaches for building mutual value. Pearson Education India.

Márquez, P., Reficco, E., y Berger, G. (2010) Negocios inclusivos: iniciativas de mercado con los pobres de Iberoamérica. In Inter-American Development Bank Felipe Herrera Library. Bogotá D.C., Colombia.

Martínez, J. C. (2008) Puntos fuertes y débiles de las cooperativas desde un concepto amplio de gobierno empresarial. REVESCO. Revista de Estudios Cooperativos, Segundo Cuatrimestre, N ${ }^{\circ}$ 95, pp. 65-93.

Mellor, J. W. (1999) Faster More Equitable Growth-The Relation Between Growth in Agriculture and Poverty.

Ministerio de Hacienda y Crédito Público. (2012) Decreto 380 de 2012. Bogotá D.C., Colombia.

Ministerio de Tecnologías de la Información y las Comunicaciones. (2018) Datos Abiertos Gobierno Digital Colombia. Retrieved April 26, 2018, from https://www.datos.gov.co/Agricultura-y-Desarrollo-Rural/Cadena-Productiva-BananoExportaciones/4k8e-ex6x/data

Nullvalue. (2011, November 11) Cooperativa Bananera Exportará a Europa. El Tiempo. 
Ostertag, C. F., Sandoval, O. A., Barona, J. F., y Mancilla, C. (2014) An Evaluation of Fairtrade Impact on Smallholders and Workers in the Banana Sector in northern Colombia. Bogotá, Colombia.

Pineda, M. A. (2015) Negocios y sostenibilidad en la Base de la Pirámide. Punto de Vista, No 5, Vol. 9.

PNUD - Programa de las Naciones Unidas para el Desarrollo. (2010) Informe sobre Desarrollo Humano 2010. Nueva York.

Porter, M. E. (1985) Competitive advantage: creating and sustaining superior performance. 1985. In New York: FreePress (Vol. 43).

Prahalad, C. K. (2005) The business opportunity at the base of the pyramid (Wharton Sc). Upper Saddle River.

Prahalad, C. K., y Hart, S. L. (2002) Negocios en la base de la pirámide. Gestión, № 7, Vol. 3, pp. 108-117.

Ramírez, A. J. R. (1999) Cooperativismo y participación en Andalucía. CIRIEC-España, Revista de Economía Pública, Social y Cooperativa, № 31, pp. 113-148.

Reficco, E. (2010) NEGOCIOS INCLUSIVOS Y RESPONSABILIDAD SOCIAL UN MATRIMONIO COMPLEJO. Debates IESA, No 15 , Vol. 3.

SENA - Servicio Nacional de Aprendizaje. (2013) Estudio de Caracterización del sector agropecuario en Colombia.

Simanis, E., y Hart, S. (2008) The base of the pyramid protocol: Toward next generation BoP strategy. Cornell University, $\mathrm{N}^{\mathrm{o}} 2$, pp. 1-57.

SNV - Servicio Holandés de Cooperación al Desarrollo, y CECODES - Consejo Empresarial Colombiano para el Desarrollo. (2008) Los Negocios Inclusivos en Colombia. Bogotá D.C., Colombia.

SNV - Servicio Holandés de Cooperación al Desarrollo, y WBCSD - Consejo Mundial Empresarial para el Desarrollo Sostenible. (2008) Negocios inclusivos. Iniciativas Empresariales Rentables con Impacto en el Desarrollo. Quito.

Van Haeringen, R., y de Jongh, W. R. (2010) Los negocios inclusivos en el sector agropecuario: práctica y desafíos. Revista Estudios Agrarios, № 44.

WBCSD - Concejo Mundial Empresarial para el Desarrollo Sostenible. (2006) Oportunidades de negocios para reducir la pobreza. Una guía de campo.

Woodhill, J. (2016) Inclusive Agribusiness: The State of Play. Bonn, Germany.

Yin, R. K. (1989) Case study research: Design and methods, revised edition. In Applied Social Research Methods Series (Vol. 5).

Zabala, H. (2007) Las cooperativas de trabajo asociado. In La fuerza de los argumentos. Programa de televisión producido por la Rectoría de la Universidad de Antioquia y el Instituto de Filosofía, transmitido por Teleantioquia el. 